\title{
Organ-preserving treatment of an epididymal abscess in a patient with spinal cord injury
}

\author{
J Pannek $^{1}$, S Pannek-Rademacher ${ }^{2}$ and M Cachin-Jus ${ }^{2}$
}

Study design: Case report.

Objectives: To describe a case of successful organ-preserving treatment of an epididymal abscess in a tetraplegic patient. Setting: Spinal Cord Injury Rehabilitation Centre in Switzerland.

Methods: We present the clinical course of a patient with an epididymal abscess caused by multiresistant bacteria. As the patient declined surgical intervention, a conservative approach was induced with intravenous antibiotic treatment. As the clinical findings did not ameliorate, adjunctive homeopathic treatment was used.

Results: Under combined treatment, laboratory parameters returned to normal, and the epididymal abscess was rapidly shrinking. After 1 week, merely a subcutaneous liquid structure was detected. Fine-needle aspiration revealed sterile purulent liquid, which was confirmed by microbiological testing when the subcutaneous abscess was drained. Postoperative course was uneventful.

Conclusions: As the risk for recurrent epididymitis is high in persons with spinal cord injury, an organ-preserving approach is justified even in severe cases. Homeopathic treatment was a valuable adjunctive treatment in the above-mentioned case. Therefore, prospective studies are needed to further elucidate the future opportunities and limitations of classical homeopathy in the treatment of urinary tract infections.

Spinal Cord (2014) 52, S7-S8; doi:10.1038/sc.2014.50

\section{INTRODUCTION}

Virtually all patients with spinal cord injury (SCI) develop neurogenic lower urinary tract dysfunction (NLUTD). The primary goal of bladder management in patients with NLUTD as a result of suprasacral SCI is to achieve low urine storage pressure ${ }^{1}$ in order to protect renal function.

Irrespective of the method chosen for bladder management, the risk for urinary tract infection (UTI) is elevated in these persons. In men with NLUTD due to SCI, especially epididymo-orchitis is a common complication, which is found in up to $38.5 \%$ of the patients. $^{2}$ If epididymo-orchitis is complicated by abscess formation, orchiectomy or epididymectomy is the method of choice. ${ }^{3}$ However, patients try to avoid ablative genital surgery whenever possible. We present the case of a multimodal approach of an organ-sparing treatment of an epididymal abscess.

\section{CASE REPORT}

In January 2014, a 35-year-old male patient with a traumatic complete tetraplegia below C6 since 2001, American Spinal Injury Association impairment scale A, was referred to us owing to a swelling of the left testicle, fever and increased spasticity. He emptied his bladder by reflex voiding five times a day. Since 5 days, he took norfloxacin without clinical improvement, long-term medication with baclofen $3 \times 10 \mathrm{mg}$.

On physical examination, the left testicle was tender and swollen; the scrotal skin was reddened and stretched. Ultrasound revealed a thickened epididymis with a large $(1.4 \times 1.3 \mathrm{~cm})$ abscess (Figure 1), confirmed by Doppler ultrasound. Urinalysis demonstrated a UTI with enterobacteria, sensitive only to imipenem, norfloxacin and nitrofurantoin.

Blood testing showed elevated leukocyte count $\left(15.78 \mathrm{ml}^{-1}\right)$ and C-reactive protein $\left(83 \mathrm{mgl}^{-1}\right)$.

Owing to the progressive inflammation despite norfloxacin treatment, intravenous antibiotic treatment with imipenem and local measures (cooling, immobilization) were initiated. As both the abscess and the blood parameters remained unchanged after 3 days, epididymectomy/orchiectomy was proposed, which was rejected by the patient. Therefore, we initiated homeopathic treatment with Hamamelis virginiana C30, three times daily for 4 days, followed by Hamamelis C 200 two times daily for another 5 days. Under homeopathic treatment, the abscess became significantly smaller within 3 days and the infection parameters were decreasing. After 4 days, the abscess was not visible any more (Figure 2); however, there was a fluid collection under the scrotal skin. Needle aspiration revealed pus, which was sterile on microbiological testing. The following day, the abscess was incised; microbiologic examination of the fluid confirmed no growth of bacteria. During surgery, no cavity in the epididymis could be detected. After 1 more week, the incision could be closed. Sonographic control demonstrated an intact testicle and epididymis.

\section{DISCUSSION}

To our knowledge, this is the first report of a successful organ-sparing treatment for an epididymal abscess in a patient with SCI. A similar 


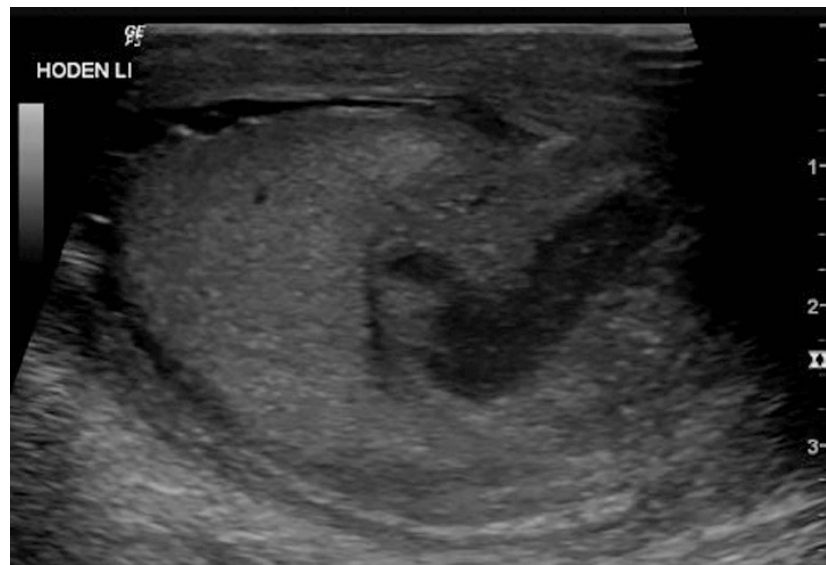

Figure 1 Ultrasound of the left scrotum: large epididymal abscess.

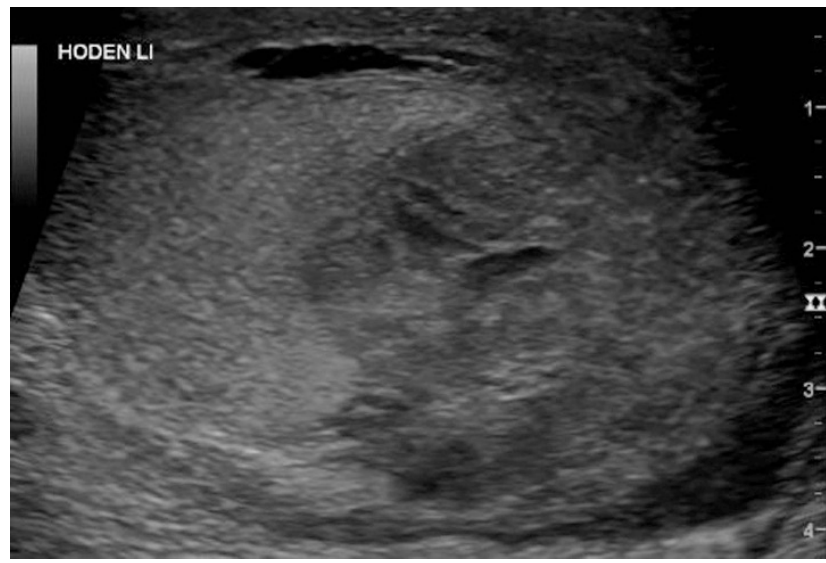

Figure 2 Ultrasound of the left scrotum under treatment, 5 days later.

case has recently been reported in a patient under immunosuppression. ${ }^{4}$ Generally, the risk to acquire an epididymitis is elevated in patients with SCI due to the NLUTD. ${ }^{2}$ The bacteria detected in the urine are usually the causative agents. The key to organ preservation is an early and intensive multimodal approach, including immobilization, cooling, long-term (at least 10 days) antibiotic treatment and low-pressure bladder drainage. In the case presented here, however, abscess formation progressed and serum infection parameters remained elevated although all mentioned measures were taken and the bacteria were susceptible to both antibiotics, which were applied for more than a week. After adjunctive homeopathic treatment, local findings improved and infection parameters returned to normal. The quick amelioration of both the clinical symptoms and the sonographic findings demonstrated that homeopathic treatment might have significantly aided in the recovery.

We used classical homeopathy. In brief, this term comprises that a single remedy was selected based on the totality of signs and symptoms of the individual patient. ${ }^{5}$ Thus, Hamamelis virginiana cannot be regarded as a general remedy for epididymal abscess formation, but the particular symptoms of the individual patients have to be taken into consideration.

\section{CONCLUSIONS}

In persons with SCI suffering from epididymo-orchitis, an organpreserving approach may be considered even in severe cases. Homeopathic treatment might have been a valuable adjunctive treatment in the mentioned case. Therefore, prospective studies are needed to further elucidate the future opportunities and limitations of classical homeopathy as an adjunct in the treatment of UTI.

\section{CONFLICT OF INTEREST}

The authors declare no conflict of interest.

1 Stöhrer M, Blok B, Castro-Diaz D, Chartier-Kastler E, Del Popolo G, Kramer G et al. EAU guidelines on neurogenic lower urinary tract dysfunction. Eur Urol 2009; 56 81-88.

2 Mirsadraee S, Mahdavi R, Moghadam HV, Ebrahimi MA, Patel HR. Epididymo-orchitis risk factors in traumatic spinal cord injured patients. Spinal Cord 2003; 41: 516-520.

3 Granados Loarca EA, Del Monte GR, Palou Redorta J, Villavicencio Mavrich H. Epididymo-testicular abscess (article in Spanish). Arch Esp Urol 1994; 47 553-556.

4 Zaid UB, Bagga HS, Reese AC, Breyer BN. Intratesticular abscess in a solitary testicle: the case for testicle sparing management. Case Rep Med 2013; 2013: 184064.

5 Bellavite P, Conforti A, Piasere V, Ortolani R. Immunology and homeopathy. 1. Historical background. Evid Based Complement Alternat Med 2005; 2: 441-452. 